\title{
Monogonont rotifers as model systems for the study of micro-evolutionary adaptation and its eco-evolutionary implications
}

\author{
Steven A. J. Declerck • Spiros Papakostas
}

Received: 20 November 2015/Revised: 7 April 2016/ Accepted: 15 April 2016/Published online: 6 June 2016

(C) The Author(s) 2016. This article is published with open access at Springerlink.com

\begin{abstract}
A better understanding of the ability of organisms to adapt to local selection conditions is essential for a better insight in their ecological dynamics. The study of micro-evolutionary adaptation and its eco-evolutionary consequences is challenging for many reasons and the choice of a suitable model organism is particularly important. In this paper, we explain why monogonont rotifers, through their unique combination of traits, are ideal study organisms for this purpose. With a literature review, we demonstrate the capacity of monogonont populations to adapt to a variety of selection conditions (e.g., salinity, food shortage, elemental limitation, and disturbance regimes) within very short-time frames and highlight some potential eco-evolutionary implications. Although monogononts are increasingly used in ecoevolution-oriented studies, their potential is still underappreciated compared to other model organisms. No doubt the high prevalence of cryptic species
\end{abstract}

Guest editors: M. Devetter, D. Fontaneto, C. D. Jersabek, D. B. Mark Welch, L. May \& E. J. Walsh / Evolving rotifers, evolving science

S. A. J. Declerck $(\bowtie) \cdot$ S. Papakostas

Department of Aquatic Ecology, Netherlands Institute of Ecology (NIOO-KNAW), Wageningen, The Netherlands e-mail: S.Declerck@nioo.knaw.nl

\section{S. Papakostas}

Division of Genetics and Physiology, Department of Biology, University of Turku, Turku, Finland complexes and the lack of genomic tools form important obstacles that may discourage researchers to work with this group. Here, we argue that none of these difficulties should prevent monogonont rotifers from becoming commonly used model organisms in micro-evolutionary studies and make suggestions for future research.

Keywords Rapid adaptation - Local adaptation . Cyclic parthenogenesis - Model organism . Population differentiation $\cdot$ Brachionus

\section{Introduction}

Traditionally ecologists tended to treat species as entities with fixed traits, mostly because rates of evolutionary adaptations are believed to be extremely slow, much slower than the rate of ecological dynamics. However, it is only in the last decade that an increasing number of ecologists have come to realize that evolutionary adaptation can be as rapid as ecological processes. Although micro-evolutionary trait shifts may seem small and insignificant in magnitude compared to the large trait changes that are documented for macro-evolutionary time scales, they may still be of considerable importance from an ecological perspective, as they may strongly determine the performance of contemporary populations and their interactions with the biotic and abiotic 
environment. Indeed, a better understanding of the ability of organisms to adapt rapidly to local selection conditions may be a key to better understanding the persistence of individual populations in a changing environment through evolutionary rescue (Bell, 2013) or the dynamics of geographic range expansions (Van Doorslaer et al., 2009; Burton et al., 2010). Rapid adaptation is also a potentially important determinant of gene flow among populations (monopolization hypothesis; De Meester et al., 2002), and is a key process in the theoretical framework of evolving metacommunities (Urban et al., 2012). Furthermore, the consequences of evolutionary changes in the phenotype of organisms may go as far as impacting the functioning of entire ecosystems (Matthews et al., 2011, 2014; Miner et al., 2012).

The study of micro-evolutionary adaptation and its eco-evolutionary consequences is challenging for many reasons. The choice of a suitable model organism is particularly crucial. Cladocerans and monogonont rotifers both share a set of traits that make them ideal model organisms for the study of microevolution in metazoans (Fussmann, 2011; Miner et al., 2012). They are relatively small and easy to handle and culture. Their short generation time allows for rapid evolutionary trait responses. Importantly, two additional features associated with their cyclic parthenogenetic reproduction distinguish them from many other metazoans and make them especially amenable for work on rapid evolution: i.e., the alternation of clonal with sexual reproduction and the tight link between sexual reproduction and the formation of diapausing eggs. Clonal reproduction permits individual genotypes to be maintained for many generations in the lab and replicated in experiments, making easy comparison of traits among genotypes possible (e.g., calculation of broad sense heritabilities, study of genotype $\mathrm{x}$ environment interactions). It also enables researchers to control for genetic variation and as such facilitates the study of (the molecular basis of) phenotypic plasticity (Jeyasingh et al., 2011) and epigenetic control mechanisms. With diapausing eggs, populations can survive adverse conditions and disperse both in space and time. A nice feature of these eggs is that they can be used to reconstruct evolutionary trajectories of natural populations through time via the 'resurrection' of genotypes that may be recovered from stratified lake sediments (Kerfoot et al., 1999; Decaestecker et al., 2007; Frisch et al., 2014).
The possibilities offered by this unique combination of traits have been intensively exploited in research on Daphnia (Miner et al., 2012). However, this is much less so in the case of monogonont rotifers. In our opinion, this is remarkable because monogonont rotifers have at least four additional advantages, especially for experimental evolution work: (1) generation times in monogononts are much shorter than in cladocerans. As a result, population level trait responses to selection regimes will potentially be much faster in monogononts (assuming equal heritability levels); (2) Monogononts easily engage in sexual reproduction under experimental conditions and sexually produced diapausing eggs have been observed to hatch very quickly, i.e., within a few days (Becks \& Agrawal, 2012; Martínez-Ruiz \& GarcíaRoger, 2015). This allows the recruitment of new genotypes within a short time span (Declerck et al., 2015). Although sexual recombination may initially slow down evolution in populations due to 'genetic slippage' (Lynch \& Deng, 1994), it strongly increases genotypic diversity in experimental populations and as such broadens the genetic basis for selection to act on (Becks \& Agrawal, 2012). As a result, short-term micro-evolutionary adaptation is not confined to clonal selection, but can additionally be fueled by sexual recombination. This is expected to be especially important in selection experiments where initial levels of genotypic diversity are limited. (3) The study of evolution in laboratory populations requires effective population sizes large enough to reduce the effect of drift. Due to their smaller body size, monogonont rotifer populations can be maintained under high densities under laboratory conditions. Populations of thousands of individuals can relatively easily be kept in volumes of only one to a few liters. As a result, selection experiments can be performed in the lab under standardized experimental conditions, and do not require large-scale outdoor facilities as is typical in cladocerans. (4) Monogononts are the only aquatic metazoans that can be kept under steady state conditions in chemostats or semi-continuous batch cultures (Walz, 1993). At steady state, the size and demographic structure of a population are constant because mortality and dilution losses are precisely compensated by food-limited population growth on a continuous basis. The maintenance of steady state in laboratory evolution experiments is a great asset for several reasons. One important advantage is that it 
reduces interference of drift effects with the selection process, drift effects that otherwise may result from genetic bottle necks associated with the typical boom and bust cycles of ordinary batch cultures. Furthermore, steady state conditions in common garden experiments permit to study the potential for ecoevolutionary feedbacks simultaneously with the assessment of local adaptation itself (Declerck et al., 2015).

This paper has the aim to provide an overview of how and to what extent monogononts have been used in studies on micro-evolutionary adaptation. First, we give a brief introduction to the different approaches used for testing micro-evolutionary adaptation in field and laboratory populations. We then proceed with a review of studies that have investigated micro-evolutionary adaptation in monogononts and its eco-evolutionary consequences. Next, we discuss the potential reasons why monogonont rotifers are relatively underexploited as study organisms compared to cladocerans like Daphnia and try to identify the major hurdles that should be addressed in order to promote the group as a model system. Finally, we give suggestions for further research.

\section{Testing the hypothesis of local adaptation using monogonont rotifers}

Theories of micro-evolutionary adaptation and approaches for its examination have been elaborately described by others (Hendry \& Kinnison, 1999; Kawecki \& Ebert, 2004), so we will limit ourselves here to a short introduction to this topic and highlight the approaches most frequently adopted in studies with monogonont rotifers. The most straightforward way of studying micro-evolutionary adaptation to local selection conditions is by comparing evolved populations with their ancestral populations. Ideally, the fitness of genotypes from both population types can be experimentally compared under identical contemporary environmental conditions (i.e., a common garden). Micro-evolutionary adaptation can be demonstrated when the evolved population has a higher fitness than the ancestral one (Fig. 1a). Repetition of fitness assays during the adaptation process may also allow a detailed study of the evolutionary trajectory and an assessment of rates of microevolutionary trait changes. Unfortunately, such an approach is often unfeasible because ancestral populations are not available for study. An alternative approach is to compare 'sister' populations with a common ancestral origin that have been exposed to habitats with different selection pressures. By exposing each of the populations to each of the habitat conditions, local adaptation can be demonstrated if populations show systematically higher fitness under the conditions of their own habitat compared to populations that have evolved under different conditions (Fig. 1b; Kawecki \& Ebert, 2004). Although this approach precludes the estimation of evolutionary rates, it allows studying adaptation along gradients of selection conditions and, in some cases, to derive rates at which populations differentiate.

The study of micro-evolutionary adaptation can be based on field as well as laboratory populations. Laboratory studies typically involve a selection experiment where replicate populations originating from the same ancestral field population are exposed to well-defined selection conditions for multiple generations. Subsequently, evolutionary adaptation is then tested in assays with a common garden design. In these assays, fitness of genotypes (in the case of monogononts: clones) is compared between 'evolved' and 'ancestral' populations (Fig. 1a) or between populations from contrasting selection conditions (Fig. 1b). Field-based studies most often have a similar approach, except that genotypes are isolated from natural populations. Given the difficulties associated with performing common garden experiments under field conditions, habitat conditions are most often mimicked in the laboratory. Both approaches have important advantages and disadvantages. Field studies have the potential of revealing how and to what extent specific environmental factors drive patterns of genetic adaptation in real-world metapopulations provided that the relevant factors causing diverging selection regimes in the field can be singled out and reproduced successfully in the laboratory. In contrast, experimental evolution studies tend to be more artificial and therefore less representative. Their major strength, however, lays in the fact that experimental selection regimes can be better controlled and replicated so that confounding factors can be avoided and chance effects minimized. They may as such serve better to provide proof of principle for the evolutionary potential of populations to specific factors, as well as facilitate more detailed and replicated 


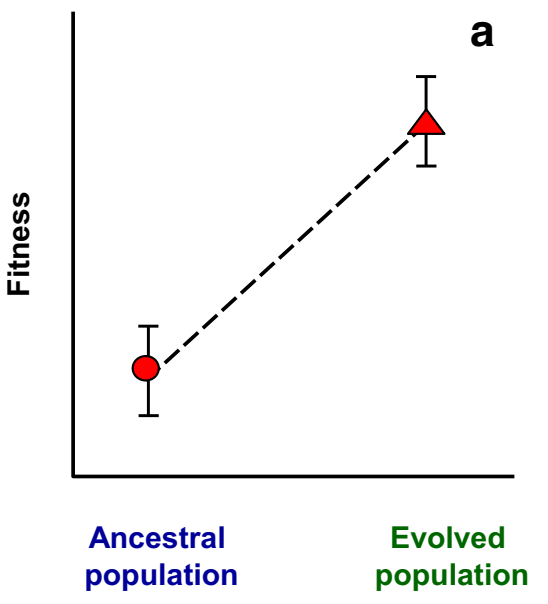

Fig. 1 Schematic representation of expected fitness reaction norms under the scenario of micro-evolutionary adaptation. a Comparison between the fitness of genotypes from evolved populations with genotypes from ancestral populations. All genotypes are exposed to the same environment representing contemporary conditions. b Fitness comparison of genotypes

investigations of the speed and temporal trajectory of micro-evolutionary adaptations.

The experimental evaluation of micro-evolutionary adaptation requires a careful choice of which trait is to be used as indicator for fitness. With that respect, consideration of the growing conditions and relevant time scale is of great importance. Being cyclic parthenogens, monogonont rotifers are able to alternate between clonal growth and sexual reproduction. Clonal growth can be very rapid because it circumvents the costs associated with sexual reproduction. Sexual reproduction typically results in the production of diapausing eggs, which give rise to genetically diverse offspring and which are needed to survive adverse environmental conditions. Despite being costly on the short term, sexual reproduction is therefore expected to be mainly advantageous in variable habitats and is, through its association with resting stages, indispensable in ephemeral habitats. As a result, in the ephemeral type of habitats, fitness needs to be considered on the long-term encompassing multiple cycles of clonal and sexual reproduction. Such 'long term' fitness can be estimated as the capacity of genotypes to produce high numbers of good quality diapausing eggs (Serra \& King, 1999; Smith \& Snell, 2012). Differences in bet hedging strategies with respect to timing of hatching, however, form an important complicating aspect hence the

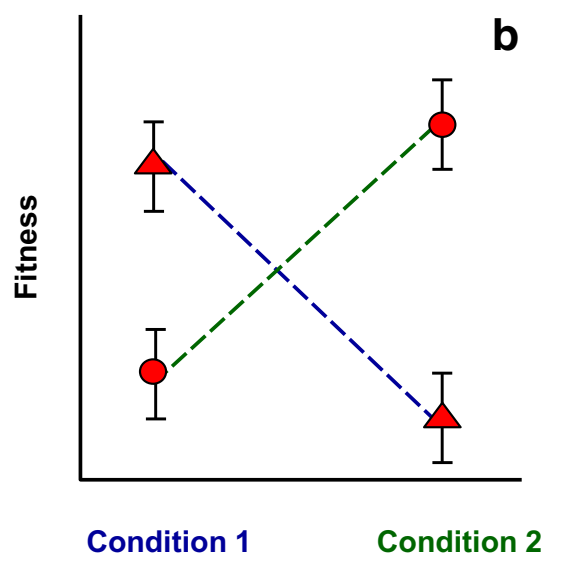

from populations with common ancestry that have evolved under contrasting selection conditions. Genotypes of both populations are exposed to each of the conditions corresponding to original selection conditions. For a more detailed discussion on different types of reaction norms and their interpretation, we refer to Kawecki \& Ebert (2004)

geometric mean of fitness across cycles is a more appropriate measure (Garcia-Roger et al., 2014). When fitness is considered within a growing season or in permanent habitats with constant and predictable environments (e.g., standardized laboratory conditions), the success of genotypes will mainly lie in their contemporary capacity to maximize relative abundance ('short term' fitness). Especially with respect to the latter, it is important to consider the type of growth conditions under which selection is taking place. If conditions select mainly for rapid growth at high food concentrations, for example due to frequent disturbances, high predation rates or experimental dilution rates, genotypes with the highest population growth rate will be favored. In contrast, conditions of low population turnover rates associated with strong resource competition will select for genotypes that are best able to reduce food levels below that of the food requirements of competing genotypes. In the first case, comparison of the intrinsic rate of population increase at saturating food conditions may be the most convenient way for evaluating the relative fitness of genotypes. In the latter case, the most straightforward measure of fitness for a genotype is its relative success in competition experiments. This may however be difficult in the absence of molecular markers that allow identification of genotypes. Alternatively, competitive ability may also be derived from 
the steady state residual food concentration $\left(\mathrm{C}^{*}\right)$ in (semi-)continuous cultures. To conclude, the choice of the right fitness parameter may thus be crucial for testing the hypothesis of micro-evolutionary adaptation. Ideally, such choice should be based on a good understanding of the relevant time scale and the growth conditions under which the investigated populations have adapted. The latter is quite straightforward for laboratory evolution experiments, but is more difficult to assess in field-based studies.

Another issue that is especially relevant for microevolutionary studies with monogonont rotifers is the existence of cryptic species. Since the discovery of cryptic species in Brachionus plicatilis (Gómez \& Serra, 1995; Gómez et al., 2002), the number of rotifer taxa known to be composed of cryptic species has steadily increased during the last decade (Walsh et al., 2009; Obertegger et al., 2012). Furthermore, several studies have provided evidence for substantial ecological segregation among such species (Gabaldón et al., 2015a; Papakostas et al., 2016). Because cryptic species are often found to be sympatric (Gómez et al., 2002; Gabaldón et al. 2015b, Papakostas et al., 2016), the potential occurrence of multiple species in experimental populations of microevolution studies may incur considerable risks. Indeed, when the species identity of genotypes is ignored, trait shifts in populations may not be unequivocally due to microevolutionary responses, but may as well be caused by changes in the relative abundance of cryptic species. We therefore stress the need of an initial molecular screening and proper identification of all candidate genotypes to be involved in experiments.

\section{Review of studies using monogononts}

We identified a total of 11 empirical studies on microevolutionary adaptation in monogononts. An overview of some main characteristics of these studies is provided in Table 1. Three studies were field-based, whereas eight studies took an experimental evolution approach. Two studies (Fussmann et al., 2003; GarciaRoger et al., 2014) were mainly theoretical. It is remarkable that only two taxa of one single genus have hitherto been considered in studies of micro-evolutionary adaptation in monogononts, i.e., the cryptic species complexes Brachionus calyciflorus Pallas 1766 and B. plicatilis Müller 1786. All field studies so far have involved the $B$. plicatilis complex whereas $B$. calyciflorus tends to be more popular in experimental studies. Whereas studies on B. plicatilis consistently checked the identity of cryptic species, this has seldom been done in studies with $B$. calyciflorus (Table 1). With the exception of one study (Scheuerl \& Stelzer, 2013), experimental studies always made use of chemostats or semi-continuous batch cultures when exposing populations to experimental selection conditions. Tests of adaptation were most often based on the evaluation of short-term fitness indicators, mainly the intrinsic population growth rate or surrogate variables (e.g., lifetime fecundity) and, more rarely, the outcome of direct competition experiments (Stelzer, 2011) or the capacity to suppress food levels (C*; Declerck et al., 2015). So far, only few studies considered evolutionary responses in long-term fitness (i.e., diapausing egg production capacity; Campillo et al., 2011; Smith \& Snell, 2012). All experiments were able to demonstrate evolutionary change within a limited amount of time, i.e., less than 125 days, providing strong evidence for the idea that monogononts have a high capacity to rapidly adapt to a changing environment.

Local adaptation to abiotic conditions and food quality

Studies on local adaptations of natural monogonont populations have so far mainly focused on adaptation to salinity. In a common garden experiment, Campillo et al. (2009) demonstrated a high evolutionary potential of natural populations with respect to salinity and temperature, as evidenced by pronounced genotype by environment interactions for fitness-related traits, such as total diapausing egg production and intrinsic population growth rate. Campillo et al. (2011) reanalyzed these data to evaluate the degree of local adaptation to above-mentioned environmental gradients by comparing reaction norms at the population level. The authors found clear patterns of genetic differentiation among populations and some circumstantial evidence for adaptation to salinity and possibly also to habitat permanence in a subset of the populations.

Alcantara-Rodriguez et al. (2012) evaluated potential adaptation to reduced salinity using three Mexican populations of the B. plicatilis species complex: two populations from saline lakes and one population from 


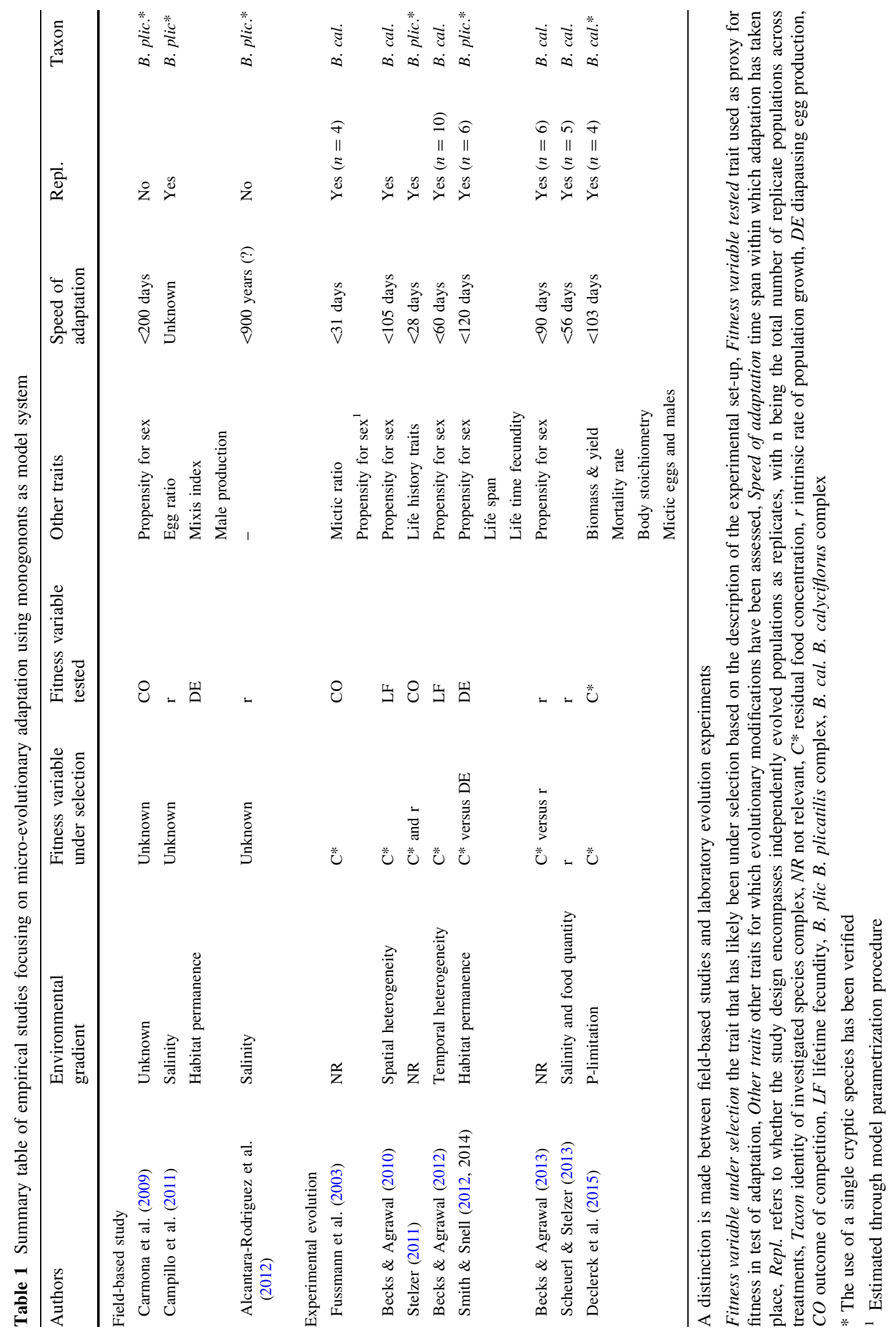


a freshwater lake. Three genotypes of each of these populations were cultured under three levels of salinity, each level corresponding to values typical for one of each lake $\left(1.1,6.5\right.$, and $9 \mathrm{~g} \mathrm{l}^{-1}$, respectively). All genotypes realized positive growth rates under highly saline conditions. In the freshwater treatment, population growth rates of all genotypes were strongly reduced, but this reduction was much more pronounced for genotypes of saltwater than of freshwater origin: population growth of the saltwater genotypes became negative, whereas freshwater genotypes were still able to realize a positive population growth. These results strongly suggest that the freshwater population of this halophile species has adapted to the conditions of the freshwater lake. It is difficult to evaluate how rapid this adaptation has developed. COI sequence data show that all of the investigated genotypes belonged to one single COI haplotype. The absence of sequence divergence suggests that the adaptation has originated after colonization of the lakes by this haplotype and that immigration rates of other haplotypes has been very limited or absent. Given the relatively slow divergence rates of the COI gene, it is unclear if this adaptation has been rapid in an eco-evolutionary sense. From a combination of geological and paleolimnological data, the rate of adaptation to freshwater conditions has been estimated to range between 24 and 900 years (Alcantara-Rodriguez et al., 2012).

Rapid adaptation to increased salinity was shown in a laboratory evolution study by Scheuerl \& Stelzer (2013) who exposed replicate identical multiclonal populations of the freshwater species B. calyciflorus to three selection conditions: two conditions representing stressful environments (high salinity and low food availability) and one condition representing 'benign' growth conditions (i.e., low salinity combined with high food availability). By design, the experimental set-up ensured selection for fast population growth and simultaneously allowed the monitoring of this fitness variable during the course of the experiment. Within 56 days, or ca. 43 clonal generations, highly saline conditions resulted in a gradual but strong fitness increase which concurred with high rates of sexual reproduction throughout the experiment. Fitness increments were less pronounced under low-quality food conditions and found to be absent under benign conditions. In addition, a common garden transplant experiment at the end of the selection experiment revealed a fitness reaction norm consistent with local adaptation.

Declerck et al. (2015) investigated micro-evolutionary adaptation of monogonont rotifers in a context of elemental limitation. They performed a replicated selection experiment subjecting multiclonal populations of $B$. calyciflorus to equal amounts of food, but with different stoichiometric qualities (phosphorus limited vs. phosphorus replete phytoplankton) under continuous culture conditions. Local adaptation of these experimental populations was studied in two consecutive common garden transplant experiments using semi-continuous cultures after approximately 100 and 200 days of selection. When fed P-limited food (LP), populations adapted to low P-food realized higher steady state biomasses, achieved higher yields and grazed down food to lower levels than did populations with a history of selection by high-P food (HP). The ability of LP-populations to persist at a lower residual concentration of P-limited food strongly suggests competitive superiority to HP-populations under P-limiting conditions. No evidence was found for adaptive changes in rotifer body elemental composition. Instead, adaptation to P-limited food was associated with reduced mortality rates and lower per capita rates of males and sexually produced diapausing eggs. Reduction in sexual investment may therefore have been an important strategy to cope with P-limitation and likely contributed to the relatively high performance of LP-adapted populations under P-limiting conditions. However, other adaptations may also have played a role, such as modifications in the functional response, efficiency of P-metabolism or other forms of homeostasis maintenance or the ability to cope with reduced biochemical quality or digestibility of P-limited algae.

Rapid evolution of sex and its implications for adaptation

Due to their cyclic parthenogenetic reproduction mode, monogonont rotifers form ideal model organisms for the study of the evolution and adaptive benefits of sex. The occurrence and evolution of sex, its fitness costs and benefits, and its adaptive significance have therefore been a major focus of microevolutionary studies on monogonont rotifers. Sex is costly for several reasons (Stelzer, 2015). Amongst others, sex reduces the number of offspring per parent 
at least by a factor of two. Sexual reproduction in monogononts also invariably results in the production of diapausing eggs that do not immediately contribute to population growth. Furthermore, recombination may break down beneficial allele combinations that have been shaped by historic selection (genetic slippage). For such reasons, stable growth conditions are expected to select against genotypes with a high propensity for sex, and several studies have provided evidence for this. Starting with multiclonal populations of B. calyciflorus in single-stage chemostat cultures, Fussmann et al. (2003) observed a strong reduction of sexual investment through time. Similarly, during the course of one growing season in a brackish pond, Carmona et al. (2009) observed a strong reduction in the mixis ratio (i.e., proportion of sexual females) of a B. plicatilis population concomitant with a genetically determined decrease in the propensity for sex. Stelzer (2011) confronted cyclic parthenogenetically reproducing strains with closely related obligatory parthenogenetic strains of $B$. calyciflorus under stable, continuous culture conditions. Especially when cyclical parthenogenetic clones invested a lot in sex, they were rapidly replaced by the obligate asexuals. In a follow-up study, Stelzer (2012) demonstrated that, compared to the cyclical parthenogens, asexual strains systematically reach higher steady state biomass and reduced residual food to lower levels. A genetics-based loss of the propensity for sex was also observed in (semi)-continuous cultures by Becks \& Agrawal (2010, 2011, 2013), Smith \& Snell (2012) and Declerck et al. (2015). However, the rate or extent of such loss was also shown to depend on the type of selection regime. Becks \& Agrawal (2013) showed that the propensity for sex was more strongly reduced in semi-continuous cultures with high compared to low dilution rates. Declerck et al. (2015) found a stronger constitutive reduction in sexual investment in populations exposed to P-limited compared to P-replete food.

Under natural conditions, however, the environment is often variable and unpredictable. Changes in environmental conditions have been shown to favor sexual reproduction in monogonont rotifers. This can be explained by the adaptive benefits of the genetic diversity generated by sexual recombination. Becks \& Agrawal (2012) showed that rates of sex evolve to higher levels during adaptation, but then decline as fitness plateaus. They also showed that sex enabled faster adaptation by generating higher fitness variability among sexual progeny, despite a reduction of average fitness (Becks \& Agrawal, 2012). For their experiment, they used populations pre-adapted to two contrasting environmental conditions. Of each population type, they continued growing replicate populations under constant conditions, while subjecting another set of these populations to the alternative regime. Populations which stayed under constant conditions showed a rather constant population density and a permanently low percentage of mictic eggs in the population. In contrast, populations exposed to changed culture conditions first responded with a decline in population density shortly followed by a strong increase in the fraction of mictic eggs (sexual investment). Population densities then recovered and the fraction of mictic eggs decreased to very low levels. A repeated assessment of propensity for sex throughout the experiment revealed a genetic basis for the observed changes in sexual investment. Furthermore, throughout the experiment, the authors monitored fitness differences between sexual and asexual offspring using separate growth rate assays (for details on the design, see Becks \& Agrawal, 2012). Sexually produced offspring had a lower mean fitness than asexually produced offspring, both in the constant as well as variable regimes. However, sex resulted in an increased fitness variance. Only when the environment was variable and during the process of selection did a fraction of the sexually produced genotypes have a higher fitness than the asexually produced genotypes. These results provide important insight in the evolutionary mechanisms that may underlie rapid adaptation in many monogonont populations and reveal the benefits of sex during adaptation, despite its considerable average fitness costs. They teach us that rapid adaptation of monogonont rotifer populations to a changing environment in continuous (i.e. non-ephemeral) habitats is not necessarily the product of selection on pre-existing clones (i.e. clonal selection) alone, but that it may also be realized by the short-term generation and proliferation of sexually produced genotypes, at least in taxa of which diapausing eggs have the capacity to hatch quickly after their production and as such contribute to the next generations within a very short time frame. During this process, sexual reproduction is favored and it will persist until fitness is maximized and the costs of sex start outweighing the benefits. From that moment on, 
selection will act against genotypes with high propensity for sex and the observed proportions of mictic females in populations will decrease. The results of Becks \& Agrawal (2012) have been corroborated by other studies as well. In an earlier study, Becks \& Agrawal (2010) demonstrated an increased propensity for sex and an increased frequency of occurrence of sexual individuals in spatially heterogeneous compared to homogenous continuous cultures. Adaptation through sustained sexual reproduction may also explain the observation by Scheuerl \& Stelzer (2013) who reported undiminished rates of sexual reproduction in B. calyciflorus populations exposed to stressful levels of salinity despite growth conditions selecting for maximum population growth rate. Similarly to Becks \& Agrawal (2012), Declerck et al. (2015) also observed high rates of sexual reproduction, but only during an episode of population decline at the start of their selection experiment. A screening of the multilocus genotype composition of these populations with microsatellite markers showed that a high diversity of newly produced genotypes quickly replaced the original clones during this period. This genotype replacement implies that sexually produced diapausing eggs must have hatched rapidly and suggest a higher fitness of at least a fraction of these newly generated genotypes.

Evolution of dormancy and related bet hedging strategies

In ephemeral habitats, production of diapausing eggs forms an essential requirement for population persistence and genotypes will be selected for this trait, provided there is sufficient heritable genetic variation. With some exceptions (Gilbert \& Schreiber, 1995), sexual reproduction and resting egg formation are tightly linked in monogononts. Selection for diapausing egg production will thus necessarily affect sexual investment, irrespective of other costs and benefits associated with sex. Based on common garden essays involving field-derived genotypes of multiple populations, Campillo et al. (2011) suggested a negative correlation between hydroperiod duration and the production of diapausing eggs. Such a relationship was indeed experimentally supported by Smith \& Snell (2012). They exposed replicate rotifer populations in chemostats to two treatments, mimicking either permanent or ephemeral habitat. Populations in 'permanent' chemostats were kept under constant growth conditions during the entire course of the experiment. Populations in 'ephemeral' cultures were reset five times over the course of 45 weeks. This was done by replacing the active populations by hatchlings derived from the diapausing eggs produced by these populations. For the rest, conditions in the chemostats were kept as similar as possible. Populations of ephemeral conditions produced higher amounts of diapausing eggs, maintained a higher propensity for sex and contained higher frequencies of mictic females compared to populations exposed to permanent conditions.

In unpredictable, ephemeral habitat, fitness is also expected to be determined by the timing of diapausing egg hatching (Spencer et al., 2001). Complete hatching of all diapausing eggs is a risky strategy in an unpredictable habitat where suitable hatching stimuli do not invariably result in a period of benign conditions that allow the successful regeneration of diapausing eggs. In such a habitat, a safer strategy (i.e. a bet hedging strategy) is to hatch only part of the diapausing eggs during each of subsequent periods of favorable conditions. Although this may come at the cost of short-term fitness, it enhances the long-term probability of persistence. Theory predicts that the optimal fraction of hatching eggs will be determined by the probability that favorable hatching conditions will result in a successful growing season. Because of its impact on long-term fitness, the bet hedging strategy can be considered a trait that is expected to be under strong selection by the predictability of the habitat. Although theoretical models allow prediction of bet hedging strategies from habitat predictability (Spencer et al., 2001; Garcia-Roger et al., 2014), straightforward empirical support for such a relationship in monogonont rotifers is so far very limited. Demonstration of such a relationship in natural populations is empirically demanding because it requires long-term field data on such variables as habitat permanence, diapausing egg deterioration, water column diapausing egg production and hatching rates (Garcia-Roger et al., 2014). Undoubtedly, an experimental evolution approach has the potential to alleviate several of these challenges. In accordance with theory, Garcia-Roger et al. (2014) reported a positive relationship between hatching fraction in diapausing eggs from natural populations and an 
assumed proxy of the permanence of their habitat (i.e. zooplankton species richness).

Potential of using monogononts to study ecoevolutionary feedbacks

We have provided ample evidence that monogonont rotifers are capable of adapting rapidly to a changing environment. In all laboratory evolution experiments, adaptation was demonstrated within less than 125 days. Such rapid adaptation may have important ecological implications. Indeed, when micro-evolutionary change takes place at time scales similar to those at which ecological dynamics play, a mutual interaction becomes possible (Hairston et al., 2005; Fussmann et al., 2007; Ellner et al., 2011) where rapid evolutionary change may alter the trajectory of ecological dynamics and vice versa. The study of such eco-evolutionary dynamics currently is a rapidly growing field of investigation and holds great promise to deepen our understanding of a wide variety of ecological phenomena in nature (Matthews et al., 2011; Schoener, 2011; Urban et al., 2012). So far, the large majority of eco-evolutionary studies involving rotifers used them as grazers of phytoplankton populations and focused on the consequences of microevolutionary change in the algae only (Yoshida et al., 2003; Becks et al., 2010). Despite the great potential of monogonont rotifers to evolve rapidly, only few ecoevolution oriented studies have employed them as focal organisms. In single-stage chemostats, Fussmann et al. (2003) observed a strong decline in the propensity of $B$. calyciflorus populations to reproduce sexually, concurrently with changes in their population dynamics. Using a mathematical model, they showed that changes in the population dynamics could only be explained upon incorporation of selection against sexual reproduction as term in their equations. Stelzer (2012) suggested that a shift from sexually to asexually reproducing $B$. calyciflorus populations, as observed by Stelzer (2011), should result in increased steady state rotifer biomass and increased grazing pressure on algae. Contrasting populations adapted to P-limited food with populations adapted to P-replete food, Declerck et al. (2015) demonstrated higher steady state biomass and lower residual food concentrations of the first compared to the latter under P-limiting food conditions. The observation of different mortality rates and propensity for sex between populations with different selection histories suggest potentially strong eco-evolutionary consequences for the demography of the rotifer populations as well as their grazing impact on primary producers.

\section{Monogononts: underappreciated opportunities}

Despite their clear advantages, especially for laboratory evolution experiments, monogononts seem to be strongly underexploited in studies of rapid adaptation and eco-evolutionary dynamics, especially when compared to cladocerans. We can only speculate about the reasons for this difference. Admittedly, there are some disadvantages related to the study of monogononts. Their relatively small size may complicate the study of several aspects, e.g. life history, elemental or biochemical composition. A high propensity for sex combined with the capacity of diapausing eggs to hatch quickly after their production often hinders the maintenance of pure genetic lines in the laboratory. The wide occurrence of poorly resolved cryptic species complexes complicates the set-up of laboratory evolution experiments and the interpretation of local adaptation in the field. Another important reason is undoubtedly the larger size of the Daphnia research community and its long tradition in evolutionary and genomic research (Miner et al., 2012), which has resulted in the availability of fully sequenced, assembled and annotated genomes (Colbourne et al., 2011) and a broad range of genomic tools. It is our opinion, however, that none of the difficulties mentioned above should prevent monogonont rotifers from becoming commonly used model organisms for evolutionary research. We would therefore call for initiatives within the rotifer community to eliminate the obstacles, for example, by undertaking concerted actions to resolve species complexes and develop reliable and easy-to-use morphological or molecular diagnostic tools that help with their identification. There is definitely the need for whole genome sequencing of members of at least a few key species. Top candidates are species belonging to the genus Brachionus. Species of this genus are particularly amenable to evolutionary research due to their ease of culture and experimental manipulation. This is exemplified by the fact that micro-evolutionary studies so far have been conducted on this genus only. The B. plicatilis complex is a strong candidate taxon 
because it is by far the most well-documented and resolved monogonont species complex. While $B$. calyciflorus has also been the subject of many studies, its species complex is yet poorly understood although efforts are currently being done to fill this knowledge gap (Papakostas et al., 2016). A major advantage of $B$. calyciflorus is that it is a common freshwater species in many parts of the world. It is therefore more widely available to researchers as study object for populationoriented studies. Being a freshwater species, it may also be more representative for monogononts than the predominantly saline $B$. plicatilis.

\section{Future perspectives}

Here, we have discussed a number of studies that have demonstrated rapid adaptation of monogonont populations to changing environmental conditions, mainly by showing evolution of fitness-related traits. However, in many cases, it remains unclear which are the specific traits that have been under selection and as such, underlie the observed fitness responses (Table 1). In-depth knowledge of the traits that are involved in adaptation is, nevertheless, key to better understand its eco-evolutionary consequences and predict its effects on ecosystem functioning. To take the example of adaptation of Brachionus rotifers to P-limitation (Declerck et al., 2015), it is yet unknown if adaptation also involves modifications at the level of food ingestion. It is reasonable to expect that the evolutionary response of rotifers to P-limitation would also involve an increased food and therefore P-uptake rate (i.e. compensatory feeding). Conversely, one could also think of an entirely opposite strategy where P-uptake efficiency is increased via the reduction of gut passage time. Grazing pressure on the algal food source and nutrient cycling would be enhanced in the former case, while the opposite would be true in the latter case. Both hypotheses can only be tested via grazing experiments. This example demonstrates that future research needs to focus more on a mechanistic understanding of observed adaptations, by identifying concurrent changes in e.g., physiology, behavior, metabolism, life history strategy. Eventually we should also strive to obtain deeper knowledge on the molecular processes and the genes involved in the adaptations. For monogononts that can be cultured in the laboratory, we advocate the combination of selection and common garden experiments with essays designed to reveal evolutionary modifications in candidate traits expected to be important in contributing to fitness in the altered environment. Such approach can be especially powerful when informed by gene expression profiling. Gene expression profiling describes the measurement of the expression level of thousands of gene products either at the mRNA level (transcriptome profiling) or at the protein level (proteome profiling). Adaptation may involve changed expression levels of those genes whose function has been targeted by selection. Accumulating evidence suggests that selection-mediated gene expression changes may be key in driving local adaptations (Brawand et al., 2011; Fraser, 2011, 2013; Enard et al., 2014). Importantly for monogonont rotifers, modern approaches of mRNA and protein quantification allow profiling even without a reference genome (e.g. Martin \& Wang 2011), while functional annotation can be readily achieved for at least a substantial fraction of quantified gene products (e.g. Primmer et al., 2013).

Rapid adaptation is most commonly attributed to selection on standing genetic variation. However, a better understanding of the link between phenotypic variation and DNA sequence variation is still needed. As we anticipate the availability of a full genome sequence of Brachionus species, the use of genome scans holds a lot of promise. For example, with costefficient methods, such as pool-GWAS (Bastide et al., 2013: Schloetterer et al., 2014), allele frequencies of single nucleotide polymorphisms can be compared among individuals from different populations and associations between genomic regions and phenotypic variation can be revealed (for a recent review for good practice of genome scans see Olivier et al., 2016). When performed correctly, genome scan methods may allow identifying genes or genomic regions that have been targeted by natural selection, and help with ascribing novel functional roles to candidate loci.

Furthermore, not much is known about the relative importance of de novo mutations or epigenetic modifications. Given that beneficial mutations are very rare, they are unlikely the driving force behind the micro-evolutionary changes reported for small experimental populations in the studies described by this review. However, the question what may be the role of mutation in much larger field populations has so far remained unaddressed. In addition, almost nothing is known about the significance of transgenerational 
epigenetic variation for monogononts. A first step in answering this question may be realized through 'evolution' experiments where single genotypes instead of genetically diverse populations are exposed to contrasting environmental conditions for multiple generations. Subsequent comparison of the performance of such populations in common garden transplant experiments would allow a first assessment of the potential role of epigenetic mechanisms. Ideally, such experiments are combined with assays designed to reveal variation at the molecular level, e.g. RNAi, DNA-methylation or histone modification screening. Although such molecular essays may not by themselves prove causality between molecular and trait variation, they may give a first indication of what molecular mechanism may be important in steering heritable epigenetic variation.

In conclusion, monogonont rotifers form an excellent model for the study of eco-evolutionary dynamics by means of rapid ecological adaptations. The adoption of an ecological genomics approach, albeit challenging for these microscopic animals, holds great promises as to formulate and test novel and exciting hypotheses for the emerging field of eco-evolutionary research.

Acknowledgments This work was partly supported by a grant to SAJD of the Division for Earth and Life Sciences (ALW) with financial aid from the Netherlands Organization for Scientific Research (NWO). SP acknowledges a grant from the Academy of Finland (No. 258048). We wish to thank K.D. Lemmen and E.M García-Roger for their valuable comments on an earlier version of this manuscript.

Open Access This article is distributed under the terms of the Creative Commons Attribution 4.0 International License (http:// creativecommons.org/licenses/by/4.0/), which permits unrestricted use, distribution, and reproduction in any medium, provided you give appropriate credit to the original author(s) and the source, provide a link to the Creative Commons license, and indicate if changes were made.

\section{References}

Alcantara-Rodriguez, J., J. Ciros-Perez, E. Ortega-Mayagoitia, C. R. Serrania-Soto \& E. Piedra-Ibarra, 2012. Local adaptation in populations of a Brachionus group plicatilis cryptic species inhabiting three deep crater lakes in Central Mexico. Freshwater Biology 57: 728-740.

Bastide, H., A. Betancourt, V. Nolte, R. Tobler, P. Stoebe, A. Futschik \& C. Schloetterer, 2013. A genome-wide, fine- scale map of natural pigmentation variation in Drosophila melanogaster. Plos Genetics 9: e1003534.

Becks, L. \& A. F. Agrawal, 2010. Higher rates of sex evolve in spatially heterogeneous environments. Nature 468: 89-92.

Becks, L. \& A. F. Agrawal, 2011. The effect of sex on the mean and variance of fitness in facultatively sexual rotifers. Journal of Evolutionary Biology 24: 656-664.

Becks, L. \& A. F. Agrawal, 2012. The evolution of sex is favoured during adaptation to new environments. Plos Biology 10: e1001317.

Becks, L. \& A. F. Agrawal, 2013. Higher rates of sex evolve under K-selection. Journal of Evolutionary Biology 26: 900-905.

Becks, L., S. P. Ellner, L. E. Jones \& N. G. J. Hairston, 2010. Reduction of adaptive genetic diversity radically alters eco-evolutionary community dynamics. Ecology Letters 13: 989-997.

Bell, G., 2013. Evolutionary rescue and the limits of adaptation. Philosophical Transactions of the Royal Society B-Biological Sciences 368: 20120080.

Brawand, D., M. Soumillon, A. Necsulea, P. Julien, G. Csárdi, P. Harrigan, M. Weier, A. Liechti, A. Aximu-Petri, M. Kircher, F. W. Albert, U. Zeller, P. Khaitovich, F. Grützner, S. Bergmann, R. Nielsen, S. Pääbo \& H. Kaessmann, 2011. The evolution of gene expression levels in mammalian organs. Nature 478: 343-348.

Burton, O. J., B. L. Phillips \& J. M. J. Travis, 2010. Trade-offs and the evolution of life-histories during range expansion. Ecology Letters 13: 1210-1220.

Campillo, S., E. M. Garcia-Roger, M. J. Carmona, A. Gómez \& M. Serra, 2009. Selection on life-history traits and genetic population divergence in rotifers. Journal of Evolutionary Biology 22: 2542-2553.

Campillo, S., E. M. Garcia-Roger, M. Jose Carmona \& M. Serra, 2011. Local adaptation in rotifer populations. Evolutionary Ecology 25: 933-947.

Carmona, M. J., N. Dimas-Flores, E. M. Garcia-Roger \& M. Serra, 2009. Selection of low investment in sex in a cyclically parthenogenetic rotifer. Journal of Evolutionary Biology 22: 1975-1983.

Colbourne, J. K., M. E. Pfrender, D. Gilbert, W. K. Thomas, A. Tucker, T. H. Oakley, S. Tokishita, A. Aerts, G. J. Arnold, M. K. Basu, D. J. Bauer, C. E. Cáceres, L. Carmel, C. Casola, J.-H. Choi, J. C. Detter, Q. Dong, S. Dusheyko, B. D. Eads, T. Froehlich, K. A. Geiler-Samerotte, D. Gerlach, P. Hatcher, S. Jogdeo, J. Krijgsveld, E. V. Kriventseva, D. Kueltz, C. Laforsch, E. Lindquist, J. Lopez, J. R. Manak, J. Muller, J. Pangilinan, R. P. Patwardhan, S. Pitluck, E. J. Pritham, A. Rechtsteiner, M. Rho, I. B. Rogozin, O. Sakarya, A. Salamov, S. Schaack, H. Shapiro, Y. Shiga, C. Skalitzky, Z. Smith, A. Souvorov, W. Sung, Z. Tang, D. Tsuchiya, H. Tu, H. Vos, M. Wang, Y. I. Wolf, H. Yamagata, T. Yamada, Y. Ye, J. R. Shaw, J. Andrews, T. J. Crease, H. Tang, S. M. Lucas, H. M. Robertson, P. Bork, E. V. Koonin, E. M. Zdobnov, I. V. Grigoriev, M. Lynch \& J. L. Boore, 2011. The ecoresponsive genome of Daphnia pulex. Science 331: 555-561.

De Meester, L., A. Gómez, B. Okamura \& K. Schwenk, 2002. The monopolization hypothesis and the dispersal-gene flow paradox in aquatic organisms. Acta Oecologica-International Journal of Ecology 23: 121-135. 
Decaestecker, E., S. Gaba, J. A. M. Raeymaekers, R. Stoks, L. Van Kerckhoven, D. Ebert \& L. De Meester, 2007. Hostparasite "Red Queen" dynamics archived in pond sediment. Nature 450: 870-873.

Declerck, S. A. J., A. R. Malo, S. Diehl, D. Waasdorp, K. D. Lemmen, K. Proios \& S. Papakostas, 2015. Rapid adaptation of herbivore consumers to nutrient limitation: eco-evolutionary feedbacks to population demography and resource control. Ecology Letters 18: 553-562.

Ellner, S. P., M. A. Geber \& N. G. Hairston, 2011. Does rapid evolution matter? Measuring the rate of contemporary evolution and its impacts on ecological dynamics. Ecology Letters 14: 603-614.

Enard, D., P. W. Messer \& D. A. Petrov, 2014. Genome-wide signals of positive selection in human evolution. Genome Research Cold Spring Harbor Lab 24: 885-895.

Fraser, H. B., 2011. Genome-wide approaches to the study of adaptive gene expression evolution Systematic studies of evolutionary adaptations involving gene expression will allow many fundamental questions in evolutionary biology to be addressed. Bioessays 33: 469-477.

Fraser, H. B., 2013. Gene expression drives local adaptation in humans. Genome Research 23: 1089-1096.

Frisch, D., P. K. Morton, P. R. Chowdhury, B. W. Culver, J. K. Colbourne, L. J. Weider \& P. D. Jeyasingh, 2014. A millennial-scale chronicle of evolutionary responses to cultural eutrophication in Daphnia. Ecology Letters 17: 360-368.

Fussmann, G. F., 2011. Rotifers: excellent subjects for the study of macro- and microevolutionary change. Hydrobiologia 662: 11-18.

Fussmann, G. F., S. P. Ellner \& N. G. Hairston, 2003. Evolution as a critical component of plankton dynamics. Proceedings of the Royal Society B-Biological Sciences 270: 1015-1022.

Fussmann, G. F., M. Loreau \& P. A. Abrams, 2007. Eco-evolutionary dynamics of communities and ecosystems. Functional Ecology 21: 465-477.

Gabaldón, C., M. Serra, M. Jose Carmona \& J. Montero-Pau, 2015a. Life-history traits, abiotic environment and coexistence: the case of two cryptic rotifer species. Journal of Experimental Marine Biology and Ecology 465: 142-152.

Gabaldón, C., M. Jose Carmona, J. Montero-Pau \& M. Serra, 2015b. Long-term competitive dynamics of two cryptic rotifer species: diapause and fluctuating conditions. Plos One 10: e0124406.

Garcia-Roger, E. M., M. Serra \& M. Jose Carmona, 2014. Bethedging in diapausing egg hatching of temporary rotifer populations: a review of models and new insights. International Review of Hydrobiology 99: 96-106.

Gómez, A. \& M. Serra, 1995. Behavioral reproductive isolation among sympatric strains of Brachionus plicatilis Muller 1786: insights into the status of this taxonomic species. Hydrobiologia 313: 111-119.

Gómez, A., M. Serra, G. R. Carvalho \& D. H. Lunt, 2002. Speciation in ancient cryptic species complexes: evidence from the molecular phylogeny of Brachionus plicatilis (Rotifera). Evolution 56: 1431-1444.

Gilbert, J. J. \& D. K. Schreiber, 1995. Induction of diapausing amictic eggs in Synchaeta pectinata. Hydrobiologia 313: $345-350$.
Hairston, N. G., S. P. Ellner, M. A. Geber, T. Yoshida \& J. A. Fox, 2005. Rapid evolution and the convergence of ecological and evolutionary time. Ecology Letters 8: 1114-1127.

Hendry, A. P. \& M. T. Kinnison, 1999. Perspective: the pace of modern life: measuring rates of contemporary microevolution. Evolution 53: 1637-1653.

Jeyasingh, P. D., A. Ragavendran, S. Paland, J. A. Lopez, R. W. Sterner \& J. K. Colbourne, 2011. How do consumers deal with stoichiometric constraints? Lessons from functional genomics using Daphnia pulex. Molecular Ecology 20: 2341-2352.

Kawecki, T. J. \& D. Ebert, 2004. Conceptual issues in local adaptation. Ecology Letters 7: 1225-1241.

Kerfoot, W. C., J. A. Robbins \& L. J. Weider, 1999. A new approach to historical reconstruction: combining descriptive and experimental paleolimnology. Limnology \& Oceanography 44: 1232-1247.

Lynch, M. \& H. W. Deng, 1994. Genetic slippage in response to sex. American Naturalist 144: 242-261.

Martin, J. A. \& Z. Wang, 2011. Next-generation transcriptome assembly. Nature Reviews Genetics. 12: 671-682.

Martínez-Ruiz, C. \& E. M. García-Roger, 2015. Being first increases the probability of long diapause in rotifer resting eggs. Hydrobiologia 745: 111-121.

Matthews, B., A. Narwani, S. Hausch, E. Nonaka, H. Peter, M. Yamamichi, K. E. Sullam, K. C. Bird, M. K. Thomas, T. C. Hanley \& C. B. Turner, 2011. Toward an integration of evolutionary biology and ecosystem science. Ecology Letters 14: 690-701.

Matthews, B., L. De Meester, C. G. Jones, B. W. Ibelings, T. J. Bouma, V. Nuutinen, J. van de Koppel \& J. OdlingSmee, 2014. Under niche construction: an operational bridge between ecology, evolution, and ecosystem science. Ecological Monographs 84: 245-263.

Miner, B. E., L. De Meester, M. E. Pfrender, W. Lampert \& N. G. Hairston, 2012. Linking genes to communities and ecosystems: Daphnia as an ecogenomic model. Proceedings of the Royal Society B-Biological Sciences 279: 1873-1882.

Obertegger, U., D. Fontaneto \& G. Flaim, 2012. Using DNA taxonomy to investigate the ecological determinants of plankton diversity: explaining the occurrence of Synchaeta spp. (Rotifera, Monogononta) in mountain lakes. Freshwater Biology 57: 1545-1553.

Olivier, F., H. Martins, K. Caye \& S. D. Schoville, 2016. Controlling false discoveries in genome scans for selection. Mol. Ecol. 25: 454-469.

Papakostas, S., E. Michaloudi, K. Proios, M. Brehm, L. Verhage, J. Rota, C. Peña, G. Stamou, V. L. Pritchard, D. Fontaneto, \& S. A. J. Declerck, 2016. Integrative taxonomy recognizes evolutionary units despite widespread mitonuclear discordance: evidence from a rotifer cryptic species complex. Systematic Biology 65: 508-524.

Primmer, C. R., S. Papakostas, E. H. Leder, M. J. Davis \& M. A. Ragan, 2013. Annotated genes and nonannotated genomes: cross-species use of gene ontology in ecology and evolution research. Molecular Ecology 22: 3216-3241.

Scheuerl, T. \& C.-P. Stelzer, 2013. Patterns and dynamics of rapid local adaptation and sex in varying habitat types in rotifers. Ecology and Evolution 3: 4253-4264. 
Schloetterer, C., R. Tobler, R. Kofler \& V. Nolte, 2014. Sequencing pools of individuals-mining genome-wide polymorphism data without big funding. Nature Reviews Genetics 15: 749-763.

Schoener, T. W., 2011. The newest synthesis: understanding the interplay of evolutionary and ecological dynamics. Science 331: 426-429.

Serra, M. \& C. E. King, 1999. Optimal rates of bisexual reproduction in cyclical parthenogens with density-dependent growth. Journal of Evolutionary Biology 12: 263-271.

Smith, H. A. \& T. W. Snell, 2012. Rapid evolution of sex frequency and dormancy as hydroperiod adaptations. Journal of Evolutionary Biology 25: 2501-2510.

Spencer, M., N. Colegrave \& S. S. Schwartz, 2001. Hatching fraction and timing of resting stage production in seasonal environments: effects of density dependence and uncertain season length. Journal of Evolutionary Biology 14: 357-367.

Stelzer, C.-P., 2011. The cost of sex and competition between cyclical and obligate parthenogenetic rotifers. American Naturalist 177: 43-53.

Stelzer, C.-P., 2012. Population regulation in sexual and asexual rotifers: an eco-evolutionary feedback to population size? Functional Ecology 26: 180-188.
Stelzer, C.-P., 2015. Does the avoidance of sexual costs increase fitness in asexual invaders? Proceedings of the National Academy of Sciences of the United States of America 112: 8851-8858.

Urban, M. C., L. De Meester, M. Vellend, R. Stoks \& J. Vanoverbeke, 2012. A crucial step toward realism: responses to climate change from an evolving metacommunity perspective. Evolutionary Applications 5: 154-167.

Van Doorslaer, W., J. Vanoverbeke, C. Duvivier, S. Rousseaux, M. Jansen, B. Jansen, H. Feuchtmayr, D. Atkinson, B. Moss, R. Stoks \& L. De Meester, 2009. Local adaptation to higher temperatures reduces immigration success of genotypes from a warmer region in the water flea Daphnia. Global Change Biology 15: 3046-3055.

Walsh, E. J., T. Schroeder, R. L. Wallace \& R. Rico-Martinez, 2009. Cryptic speciation in Lecane bulla (Monogononta: Rotifera) in Chihuahuan desert waters. International Association of Theoretical and Applied Limnology 30: 1046-1050.

Walz, N., 1993. Plankton Regulation Dynamics. Springer, Berlin.

Yoshida, T., L. E. Jones, S. P. Ellner, G. F. Fussmann \& N. G. Hairston, 2003. Rapid evolution drives ecological dynamics in a predator-prey system. Nature 424: 303-306. 